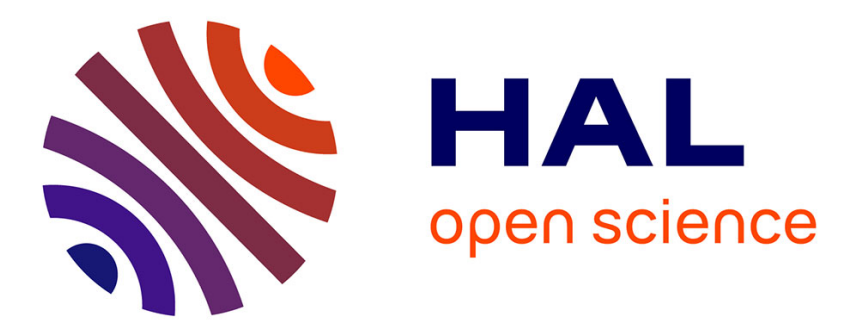

\title{
A VERSION OF WATSON LEMMA FOR LAPLACE INTEGRALS AND SOME APPLICATIONS
}

\author{
Stanislas Kupin, Sergey Naboko
}

\section{To cite this version:}

Stanislas Kupin, Sergey Naboko. A VERSION OF WATSON LEMMA FOR LAPLACE INTEGRALS

AND SOME APPLICATIONS. 2020. hal-02872226

\section{HAL Id: hal-02872226 \\ https://hal.science/hal-02872226}

Preprint submitted on 17 Jun 2020

HAL is a multi-disciplinary open access archive for the deposit and dissemination of scientific research documents, whether they are published or not. The documents may come from teaching and research institutions in France or abroad, or from public or private research centers.
L'archive ouverte pluridisciplinaire HAL, est destinée au dépôt et à la diffusion de documents scientifiques de niveau recherche, publiés ou non, émanant des établissements d'enseignement et de recherche français ou étrangers, des laboratoires publics ou privés. 


\title{
A VERSION OF WATSON LEMMA FOR LAPLACE INTEGRALS AND SOME APPLICATIONS
}

\author{
S. KUPIN AND S. NABOKO
}

\begin{abstract}
Let $f: \mathbb{R}_{+} \rightarrow \mathbb{C}$ be a bounded measurable function. Suppose that $f(t) \rightarrow 0$ at logarithmic (or $k$-logarithmic) rate as $t \rightarrow 0+$. We consider the Laplace integral of the function $f$, i.e.,

$$
I_{n}=\int_{0}^{\infty} f(t) e^{-n t} d t
$$

and obtain its asymptotics for $n \rightarrow+\infty$, which is a version of the classical Watson's lemma for the integral. Actually, the result is proved for a larger class of "slowly oscillating" functions satisfying some mild regularity conditions.
\end{abstract}

\section{INTRODUCTION}

One cannot over-estimate the rôle of asymptotic analysis in modern mathematics. We quote a short presentation of the topic from the book by Bleistein-Handelsman [1, p. vii]: "Asymptotic analysis is that branch of mathematics devoted to the study of the behavior of functions in particular limits of interest. ... Although the subject matter might appear narrow at first glance, in actuality its scope is quite large, and it is particularly relevant to applied mathematics. Indeed, the solutions to a large class of applied problems can, by means of integral transforms, be represented by definite integrals. Exact numerical values are often difficult to obtain from such representations, in which event one must resort to the method of approximation."

A particular aspect of the described activity is the study of asymptotic behavior of Laplace-type integrals, and it is also the theme of the present article. Probably, one of the most classical results in this direction is the so-called Watson's lemma, see Watson [7, 1918]. We give its simplest formulation in the "real" case (i.e., the asymptotic behavior of the integral is studied for the real values of the parameter).

Before going to the formulation of the lemma, we recall some basic notions of asymptotic analysis. Let $f, g: I:=[a, b] \rightarrow \mathbb{C}$ be two functions, and $t_{0} \in$ $(a, b),-\infty \leq a<b \leq+\infty$. First, we write $f(t)=O(g(t))$ as $t \rightarrow t_{0}$ iff

$$
|f(t)| \leq C|g(t)|, \quad t \rightarrow t_{0} .
$$

Second, $f(t)=o(g(t))$ for $t \rightarrow t_{0}$, iff

$$
\lim _{t \rightarrow t_{0}} \frac{|f(t)|}{|g(t)|}=0 .
$$

Of course, the above definitions are rewritten correspondingly when $t_{0}=a, b$ (i.e., for $t_{0}=a$, we take $t \rightarrow a+0$ instead of $t \rightarrow t_{0}$, etc.). Third, let $t_{0}=a$ and $\left\{a_{m}\right\}_{m \in \mathbb{Z}_{+}}, a_{m} \geq 0$, be a (strictly) increasing sequence. We say that $f$ admits an asymptotic expansion

$$
f(t) \sim \sum_{m=0}^{\infty} c_{m}\left(t-t_{0}\right)^{a_{m}}, \quad c_{m} \in \mathbb{C},
$$

2010 Mathematics Subject Classification. Primary: 47B35; Secondary: 30H20, 42C10. 
on the right neighborhood of $t_{0}$ (i.e., $t \rightarrow t_{0}+0$ ), iff for any natural $N$

$$
f(t)=\sum_{m=0}^{N} c_{m}\left(t-t_{0}\right)^{a_{m}}+o\left(\left(t-t_{0}\right)^{a_{N}}\right) .
$$

The natural rewriting of $(0.1)$ for $t_{0}=b$ or $t_{0} \in(a, b)$ is also obvious. Notice that the above definition makes a perfect sense even if the series $\sum_{m=0}^{\infty} c_{m}\left(t-t_{0}\right)^{a_{m}}$ is divergent.

These definitions at hand, let $f: \mathbb{R}_{+} \rightarrow \mathbb{C}$ be a locally integrable function satisfying two conditions:

(1) there is an $a>0$ such that $|f(t)|=O\left(e^{a t}\right)$, where $t \rightarrow+\infty$,

(2) we have

$$
f(t) \sim \sum_{m=0}^{\infty} c_{m} t^{a_{m}}, \quad t \rightarrow 0+
$$

where $\left(a_{m}\right)_{m \in \mathbb{Z}_{+}}, a_{m} \geq 0$, is a (strictly) increasing sequence.

The original version of the below theorem is in Watson [7, 1918], see also BleisteinHandelsman [1, Thm. in Sect. 4.1], Simon [6, Sect. 15.2] for a modern presentation.

Theorem 0.1 (Watson's lemma). Let the function $f$ satisfy above conditions (1),

(2). Consider the integral

$$
I_{n}=\int_{0}^{\infty} e^{-n t} f(t) d t
$$

Then

$$
I_{n} \sim \sum_{m=0}^{\infty} \frac{c_{m} \Gamma\left(a_{m}+1\right)}{n^{a_{m}+1}}, \quad n \rightarrow+\infty,
$$

where $\Gamma($.$) is the Euler gamma-function.$

This result is widely used for obtaining explicit asymptotics of Laplace integrals. It is quite natural that it is declined in a number of different versions and generalizations. For instance, the asymptotics of integral (0.3) in certain angular regions of complex plane are given in Copson [2], Simon [6] and Wong [8]; this is the so-called "complex case" of the lemma. Bleistein-Handelsman [1, Sect. 8.2, 8.3] and Simon [6, Thm. 15.2.2] contain also the generalization of the result to several dimensions. In Bleistein-Handelsman [1, Ch. 4,5], Watson's lemma is viewed as a rather particular case of more general results on Mellin transforms in the complex plane.

The point is that all versions of Watson's lemma we are acquainted of, deal with the case when the function $f$ admits a power asymptotic expansion on the (right) neighborhood of point $t_{0}=0$, see $(0.2)$. The powers appearing in this expansion do not need to be necessarily natural, see [6, Thm. 15.2.7] and [8, Thms in Sect. I.5].

We are interested in a substantially different situation, where the function $f$ admits an expansion of powers of logarithmic-type functions looking, for instance, as

$$
f(t) \sim \sum_{m=0}^{\infty} c_{m}\left(\frac{1}{\log _{* k}(1 / t)}\right)^{a_{m}}, \quad t \rightarrow 0+,
$$

see (2.2) for the definition of the function $\log _{* k}$. Clearly, the terms of this asymptotic expansion decay much slower than the terms of the "classical" expansion (0.2). We were unable to find the results of this kind in the literature, see $[1,2,3,4,6,8]$, nor there seems to be a straightforward way to derive them from known claims. 
This is the first reason for writing this short note for us. The second reason is an interesting application of obtained results to the study of compact Toeplitz operators on Bergman spaces and banded (Jacobi) matrices on Hilbert spaces, see KoitaKupin-Naboko-Touré [5] in this connection. Hence, the problem is to understand if there is a counterpart of Watson's results for functions $f$ admitting expansions similar to $(0.5)$ at $t_{0}=0$.

We present a large class of functions satisfying rather mild conditions for which a counterpart of Theorem 0.1 holds. In a quite interesting manner, the terms of asymptotic expansion of these functions can decay to zero at $t_{0}=0$ very slowly. For instance, the above asymptotic expansions (0.5) in terms of $\log _{* k}$ are admissible from this point of view.

We need one more definition now. Suppose that $f, g: I \times J:=[a, b] \times[c, d] \rightarrow$ $\mathbb{R}$ are (positive) functions depending on two variables $(x, t) \in I \times J$. We write $f(x,)=.\diamond(g(x,))=.\diamond_{t}(g(x,)$.$) , iff there is a constant C$ with the property

$$
|f(x, t)| \leq C|g(x, t)|
$$

for all $(x, t) \in I \times J$. Saying this a bit differently, $f(x,$.$) is O$-function of $g(x,$. uniformly in $t$. The variable of uniform dependence is sometimes indicated as a sub-index of the symbol $\diamond$ (i.e., $\diamond_{t}$ in the present situation).

Let $\varphi: \mathbb{R}_{+} \rightarrow \mathbb{C}$ be a measurable function. Suppose it is positive in a right neighborhood of 0 and it meets the following

\section{Assumptions:}

A1: $\varphi \in L^{\infty}\left(\mathbb{R}_{+}\right)$, and $\lim _{x \rightarrow 0+} \varphi(x)=0$,

A2: there are $\delta_{0}=\delta_{0}(\varphi)>0$ and $\epsilon_{0}=\epsilon_{0}(\varphi)>0$ such that for any $0 \leq \epsilon<\epsilon_{0}$ we have

$$
\varphi\left(x^{1+\epsilon}\right)=\varphi(x)(1+\diamond(\epsilon))=\varphi(x)\left(1+\diamond_{x}(\epsilon)\right),
$$

where $0 \leq x<\delta_{0}$.

A3:

$$
\lim _{x \rightarrow 0+} \frac{\log \varphi(x)}{\log x}=0
$$

Remark 0.2. A simple change of variables in (0.6) shows that assumption (A2) holds for negative $\epsilon,-\epsilon_{0}<\epsilon \leq 0$ as well.

Remark 0.3. A rather simple scaling argument gives that assumptions (A1) and (A2) yield assumption (A3). More precisely, assuming that $\left|\diamond_{x}(\epsilon)\right| \leq C_{0} \epsilon$ in (0.6), we have

in a right neighborhood of the point $x_{0}=0$.

$$
C(\log (1 / x))^{-C_{0}} \leq \varphi(x)
$$

Roughly speaking, assumption (A2) above says that the function $\varphi$ is "slowly oscillating" when its argument changes in power scale. Condition (A3) claims that $\varphi(x)$ tends to 0 when $x \rightarrow 0+$ slower than any power $x^{\alpha}, \alpha>0$. We prefer to keep assumption (A3) "as it is" for the transparency of presentation.

We are interested in the asymptotic behavior of the following Laplace integral

$$
I_{n}:=\int_{0}^{\infty} e^{-n y} \varphi(y) d y .
$$

Theorem 0.4. Let $\varphi$ be a function satisfying above conditions (A1)-(A3). We have

$$
I_{n}=\frac{1}{n} \varphi\left(\frac{1}{n}\right)(1+o(1)), \quad n \rightarrow+\infty .
$$

It goes without saying that assumption (A1) on the function $\varphi$ can be weakened. Namely, we can replace it with the following relation: 
A1': the function $\varphi$ is locally bounded; for a fixed $a>0, \varphi(x)=O\left(e^{a x}\right)$ as $x \rightarrow+\infty$ and $\lim _{x \rightarrow 0+} \varphi(x)=0$ exists.

One can prove a counterpart of Theorem 0.1 in the scale of powers of $\varphi$ obeying conditions (A1)-(A3).

Theorem 0.5 (a version of Watson's lemma). Let $\varphi$ be as in (A1)-(A3) and $f$ : $\mathbb{R}_{+} \rightarrow \mathbb{C}$ be a locally integrable function having the properties:

(1) there is an $a>0$ such that $|f(t)|=O\left(e^{a t}\right)$ for $t \rightarrow+\infty$,

(2) we have

$$
f(t) \sim \sum_{m=0}^{\infty} c_{m} \varphi(t)^{a_{m}}, \quad t \rightarrow 0+
$$

where $\left\{a_{m}\right\}_{m \in \mathbb{Z}_{+}}, a_{m} \geq 0$, is a (strictly) increasing sequence.

Then

$$
I_{n}=\int_{0}^{\infty} e^{-n t} f(t) d t \sim \sum_{m=0}^{\infty} \frac{c_{m}}{n} \varphi\left(\frac{1}{n}\right)^{a_{m}}, \quad n \rightarrow+\infty .
$$

The passing from Theorem 0.4 to Theorem 0.5 is quite elementary and follows closely the proof of $[1, \mathrm{Thm}$. in Sect. 4.1]. It uses the crucial fact that assumption (A2) is invariant with respect to the transformation $\varphi \mapsto \varphi^{\gamma}, \gamma>0$.

The meaningful constants are numbered as $C_{1}, C_{2}$, etc. Inessential constants are denoted by $c, C$ and change from one relation to another.

\section{Proof of Theorem 0.4}

Consider integral (0.8). We start making the change of variable $y=t / n$. We take two parameters $0<\eta \ll 1$ and $0<\epsilon<\epsilon_{0}$ and fix them. We shall have $\epsilon=\epsilon(\eta)$ and their choice will be made precise later, see (1.9). The integral $I_{n}$ is divided in three pieces as

$$
\begin{aligned}
I_{n} & =\frac{1}{n} \int_{0}^{\infty} e^{-t} \varphi(t / n) d t=\frac{1}{n}\left\{J_{1 n}+J_{2 n}+J_{3 n}\right\} \\
& :=\frac{1}{n}\left\{\int_{0}^{n^{-\epsilon}} e^{-t} \varphi(t / n) d t+\int_{n^{-\epsilon}}^{n^{\epsilon}} e^{-t} \varphi(t / n) d t+\int_{n^{\epsilon}}^{\infty} e^{-t} \varphi(t / n) d t .\right\}
\end{aligned}
$$

Condition (A3) (see (0.7)) implies that

$$
\frac{\log \varphi(x)}{\log x}=c(x), \quad \lim _{x \rightarrow 0+} c(x)=0,
$$

or $\varphi(x)=x^{c(x)}$ for $x \rightarrow 0+$. Consequently, for a $0<\epsilon<\epsilon_{0}$ there is a $\delta=\delta(\epsilon)>0$ such that $0 \leq c(x)<\epsilon$ for $x \in[0, \delta)$. It is convenient to choose $0<\delta<\delta_{0}$, see (0.6). In particular, there is an $N_{0}=N_{0}(\epsilon)$ large enough so that for $n \geq N_{0}$ we have $1 / n^{1-\epsilon}<\delta$. In particular, $1 / n<1 / n^{1-\epsilon}<\delta$, and so $0 \leq c(1 / n)<\epsilon$. Hence we see that

$$
\varphi(1 / n)=(1 / n)^{c(1 / n)} \geq(1 / n)^{\epsilon} .
$$

For the integral $J_{3 n}$ and $n \geq N_{0}$, we obtain

$$
\begin{aligned}
\left|J_{3 n}\right| & =\left|\int_{n^{\epsilon}}^{\infty} e^{-t} \varphi(t / n) d t\right| \leq\|\varphi\|_{L^{\infty}\left(\mathbb{R}_{+}\right)} \int_{n^{\epsilon}}^{\infty} e^{-t} d t \\
& =\|\varphi\|_{L^{\infty}\left(\mathbb{R}_{+}\right)} e^{-n^{\epsilon}} \leq\|\varphi\|_{L^{\infty}\left(\mathbb{R}_{+}\right)} \frac{1}{\left(n^{2 \epsilon} / 2 !\right)} \\
& =2\|\varphi\|_{L^{\infty}\left(\mathbb{R}_{+}\right)} \varphi(1 / n)^{2}=\varphi(1 / n) \cdot R_{3 n},
\end{aligned}
$$


where $R_{3 n}:=2\|\varphi\|_{L^{\infty}\left(\mathbb{R}_{+}\right)} \varphi(1 / n) \rightarrow 0$ as $n \rightarrow+\infty$. Pick $N_{3}=N_{3}(\varphi, \eta)$ so that we get for $n \geq N_{3}$

$$
R_{3 n}=2\|\varphi\|_{L^{\infty}\left(\mathbb{R}_{+}\right)} \varphi(1 / n)<\eta / 6 .
$$

Set $N_{3}^{\prime}=N_{3}^{\prime}(\varphi, \epsilon, \eta):=\max \left\{N_{0}, N_{3}\right\}$.

Again, we suppose $n \geq N_{0}$ defined above, so that relation (1.3) holds. We have for the bound on $J_{1 n}$

$$
\begin{aligned}
\left|J_{1 n}\right| & =\left|\int_{0}^{n^{-\epsilon}} e^{-t} \varphi(t / n) d t\right| \leq \frac{1}{n^{\epsilon}} \cdot \sup _{\tau \in\left(0, n^{-(1+\epsilon)}\right)}|\varphi(\tau)| \\
& \leq \frac{1}{n^{\epsilon}} \cdot \sup _{\tau \in(0,1 / n)}|\varphi(\tau)| \leq \varphi(1 / n) \cdot R_{1 n},
\end{aligned}
$$

where $R_{1 n}:=\sup _{\tau \in(0,1 / n)}|\varphi(\tau)| \rightarrow 0$ as $n \rightarrow+\infty$ by property (1) of the function $\varphi$. We pick $N_{1}=N_{1}(\varphi, \eta)$ so that $R_{1 n}<\eta / 6$ for $n \geq N_{1}$. Set $N_{1}^{\prime}=N_{1}^{\prime}(\varphi, \epsilon, \eta):=$ $\max \left\{N_{0}, N_{1}\right\}$.

The considerations pertaining to the bound on $J_{2 n}$ are slightly more involved. Indeed, for $t \in\left[n^{-\epsilon}, n^{\epsilon}\right]$, and $x=t / n$, we have $x \in\left[n^{-1-\epsilon}, n^{-1+\epsilon}\right]$. That is,

$$
x=\frac{1}{n}\left(\frac{1}{n}\right)^{\gamma},
$$

where $\gamma=\gamma_{x} \in[-\epsilon, \epsilon]$. For $x=t / n$ and $n \geq N_{0}$, we infer

$$
x=\frac{t}{n}=\left(\frac{1}{n}\right)^{1+\gamma} \leq n^{-(1-\epsilon)}<\delta<\delta_{0},
$$

so that $x=t / n \in[0, \delta), \delta<\delta_{0}$. Here, we used the definitions of $N_{0}$ and $\delta$, see two lines above relation (1.3). Condition (A2) on $\varphi$ (see (0.6)) implies that

$$
\begin{aligned}
\varphi(x) & =\varphi(t / n)=\varphi(1 / n)\left(1+\diamond_{t}(\gamma)\right)=\varphi(1 / n)\left(1+\diamond_{t}(\epsilon)\right) \\
& =\varphi(1 / n)\left(1+\psi_{\epsilon}(t)\right),
\end{aligned}
$$

and $\left|\psi_{\epsilon}(t)\right| \leq C_{1} \epsilon$ for $t \in\left[0, n^{\epsilon}\right)$. Here, the constant $C_{1}$ depends on $\delta_{0}$, but not on $t$. We continue as

$$
\begin{aligned}
\left|J_{2 n}-\varphi(1 / n)\right| & =\left|\int_{n^{-\epsilon}}^{n^{\epsilon}} e^{-t} \varphi(t / n) d t-\varphi(1 / n)\right| \\
& =\left|\int_{n^{-\epsilon}}^{n^{\epsilon}} e^{-t} \varphi(1 / n)\left[1+\psi_{\epsilon}(t)\right] d t-\varphi(1 / n)\right| \\
& \leq\left|\varphi(1 / n)\left(\int_{n^{-\epsilon}}^{n^{\epsilon}} e^{-t} d t-1\right)\right|+\left|\varphi(1 / n) \int_{n^{-\epsilon}}^{n^{\epsilon}} e^{-t} \psi_{\epsilon}(t) d t\right| \\
& \leq \varphi(1 / n)\left\{\left|e^{-n^{-\epsilon}}-e^{-n^{\epsilon}}-1\right|+C_{1} \epsilon\right\} \\
& \leq \varphi(1 / n) \cdot R_{2 n}
\end{aligned}
$$

where

$$
R_{2 n}:=\left\{\left|e^{-n^{-\epsilon}}-1\right|+e^{-n^{\epsilon}}+C_{1} \epsilon\right\} .
$$

For a given $\eta>0$, we fix $0<\epsilon<\epsilon_{0}$ so that

$$
C_{1} \epsilon<\eta / 6 \text {. }
$$

Choose also $N_{2}=N_{2}(\varphi, \epsilon)$ with the property $R_{2 n}<\eta / 3$ for $n \geq N_{2}$. As before, set $N_{2}^{\prime}=N_{2}^{\prime}(\varphi, \epsilon, \eta)=\max \left\{N_{0}, N_{2}\right\}$.

Putting together computations (1.4)-(1.8), we obtain

$$
\left|I_{n}-\frac{1}{n} \varphi\left(\frac{1}{n}\right)\right| \leq \frac{1}{n} \varphi\left(\frac{1}{n}\right)\left(R_{1 n}+R_{2 n}+R_{3 n}\right) .
$$


Now, let a $\eta>0$ be given. Take $\epsilon>0$ defined by (1.9) and $\delta=\delta(\epsilon)$ defined in the line following (1.2). Considering $n \geq M:=\max \left\{N_{1}^{\prime}, N_{2}^{\prime}, N_{3}^{\prime}\right\}$, see the lines below relations $(1.5),(1.6),(1.9)$, we see

$$
\left(R_{1 n}+R_{2 n}+R_{3 n}\right)<\eta .
$$

The theorem is proved.

\section{Some COROLlaries}

Take a natural $k>0$. Define the function $\exp _{* k}: \mathbb{R} \rightarrow \mathbb{R}_{+}$as

$$
\exp _{* k} x=\underbrace{\exp \ldots \exp }_{k} x, \quad x \in \mathbb{R},
$$

and

$$
\log _{* k} x:= \begin{cases}\underbrace{\log \ldots \log x,}_{k} & x>\exp _{* k} 1, \\ 0, & 0<x \leq \exp _{* k} 1 .\end{cases}
$$

It is plain that $\log _{* k}\left(\exp _{* k}(x)\right)=x, x \in \mathbb{R}$, and $\exp _{* k}\left(\log _{* k}(x)\right)=x, x>\exp _{* k} 1$. For brevity, we call these functions $k$-exp-function and $k$-log-function, respectively.

Lemma 2.1. Set $l_{k}:=\exp _{* k} 1$, the $k$-log-function satisfies assumption (A2) on the half-axis $L_{k}:=\left[l_{k},+\infty\right)$, see $(0.6)$.

Proof. The proof is simple, but we sketch it briefly for the completeness of the presentation. Indeed, the claim is clear for $k=1$. For $k=2$ and $x>l_{2}$, we have

$$
\begin{aligned}
\log _{* 2}\left(x^{1+\epsilon}\right) & =\log ((1+\epsilon) \log x)=\log \log x+\log (1+\epsilon) \\
& =\log \log (x)\left(1+\frac{\log (1+\epsilon)}{\log \log (x)}\right)=\log _{* 2}(x)\left(1+\frac{\log (1+\epsilon)}{\log _{* 2}(x)}\right),
\end{aligned}
$$

where we use that the function $\left(\log _{* 2} x\right)^{-1}$ is uniformly bounded by one on $L_{2}$ and $\log (1+\epsilon) \leq \epsilon, \epsilon \geq 0$.

We also write down the computation for $k=3$. Indeed, for $x \in L_{3}$

$$
\begin{aligned}
\log _{* 3}\left(x^{1+\epsilon}\right) & \left.=\log _{* 2}((1+\epsilon) \log x)\right)=\log (\log \log x+\log (1+\epsilon)) \\
& =\log (\log \log x[1+\log (1+\epsilon) / \log \log x]) \\
& =\log _{* 3} x\left(1+\frac{\log \left[1+\log (1+\epsilon) / \log _{* 2} x\right]}{\log _{* 3} x}\right) \\
& :=\log _{* 3} x\left(1+R_{* 3, x}(\epsilon)\right) .
\end{aligned}
$$

Once again, the function $\left(\log _{* 3} x\right)^{-1}$ is uniformly bounded by one and $R_{* 3, x}=\diamond_{x}(\epsilon)$ for $x \in L_{3}$.

For the general $k \in \mathbb{N}$, we obtain by induction

$$
\log _{* k}\left(x^{1+\epsilon}\right)=\log _{* k} x\left(1+R_{* k, x}(\epsilon)\right),
$$

where

$$
R_{* k, x}(\epsilon)=\frac{\log \left(1+\frac{\log \left(1+\ldots+\left[\frac{1+\log (1+\epsilon)}{\log _{* 2} x}\right]\right)}{\log _{* k-1} x}\right)}{\log _{* k} x},
$$

and, as before, the function $R_{* k, x}=\diamond_{x}(\epsilon)$ satisfies condition (A2), see (0.6).

For a natural $k>0$ and a $\gamma>0$, it is convenient to set

$$
\varphi_{k, \gamma}(t):= \begin{cases}\left(\frac{1}{\log _{* k}(1 / t)}\right)^{\gamma}, & 0<t \leq 1 / l_{k}, \\ 0, & t>1 / l_{k} .\end{cases}
$$


Observe that the function $\varphi_{k, \gamma}$ obeys relations (A1)-(A3). Indeed, $\varphi_{k, \gamma}$ satisfies trivially conditions (A1), (A3). For any $\epsilon>0$, the function $\varphi(x)=\log _{* k}(x)$ satisfies relation (0.6) on $L_{k}$ (i.e., on the left neighborhood of $x_{0}=+\infty$ ) by Lemma 2.1. It is bounded from above on $L_{k}$. This yields that $\varphi^{-\gamma}, \gamma>0$, satisfies relation $(0.6)$ as well. For $x \in L_{k}:=\left[l_{k},+\infty\right)$, we put $t:=1 / x \in\left(0,1 / l_{k}\right)$ and we obtain that $\varphi_{k, \gamma}$, see $(2.3)$ above, obeys $(\mathrm{A} 2)$ on $\left(0,1 / l_{k}\right)$ (i.e., on the right neighborhood of $\left.t_{0}=0\right)$.

The following corollaries follow at once from Theorem 0.4.

Corollary 2.2. For $a \gamma>0$, we have

$$
\int_{0}^{\infty} e^{-n t} \varphi_{k, \gamma}(t) d t=\int_{0}^{1 / l_{k}} e^{-n t} \log _{* k}(1 / t)^{-\gamma} d t=\frac{1}{n}\left(\log _{* k}(n)\right)^{-\gamma}(1+o(1))
$$

Corollary 2.3. For $a \gamma>0$, we have

$$
\begin{aligned}
& \int_{0}^{1} r^{n} \frac{1}{(1+\log (1 /(1-r)))^{\gamma}} d r=\frac{1}{n} \frac{1}{(\log n)^{\gamma}}(1+o(1)), \\
& \int_{0}^{1} r^{n} \frac{1}{\left(1+\log _{* k}(1 /(1-r))\right)^{\gamma}} d r=\frac{1}{n} \frac{1}{\left(\log _{* k} n\right)^{\gamma}}(1+o(1)) .
\end{aligned}
$$

Proof. We give the proof of the first claim of the corollary, the second one is completely analogous. The obvious point is to reduce the first relation to (2.4). Let $\delta>0$ be small enough. Make the change of variable $r=e^{-t}, t \in(0,+\infty)$,

$$
\begin{aligned}
\int_{0}^{1} r^{n} \frac{1}{(1+\log (1 /(1-r)))^{\gamma}} d r & =\int_{0}^{\infty} e^{-(n+1) t} \frac{1}{\left(1+\log \left(1 /\left(1-e^{-t}\right)\right)^{\gamma}\right.} d t \\
& =\int_{0}^{\delta} \cdots+\int_{\delta}^{\infty} \cdots
\end{aligned}
$$

Clearly, the second integral goes to zero as fast as $O\left(e^{-n \delta}\right)$ for $n \rightarrow+\infty$, since the function

$$
f(t)=\frac{1}{\left(1+\log \left(1 /\left(1-e^{-t}\right)\right)^{\gamma}\right.}
$$

is uniformly bounded on $\mathbb{R}_{+}$. For the first integral, an easy computation gives

$$
\begin{aligned}
& \left|\int_{0}^{\delta} e^{-(n+1) t} \frac{1}{\left(1+\log \left(1 /\left(1-e^{-t}\right)\right)^{\gamma}\right.} d t-\int_{0}^{\delta} e^{-(n+1) t} \frac{1}{(1+\log (1 / t))^{\gamma}} d t\right| \\
& \quad \leq \frac{C}{n} \frac{1}{(\log n)^{\gamma+1}}=o\left(\frac{1}{n(\log n)^{\gamma}}\right)
\end{aligned}
$$

where $C=C(\delta)$. Consequently, it remains to compute the asymptotics of the integral

$$
\int_{0}^{\delta} e^{-(n+1) t} \frac{1}{(1+\log (1 / t))^{\gamma}} d t
$$

As in Corollary 2.2, the function

$$
\psi(t)=\frac{1}{(1+\log (1 / t))^{\gamma}}
$$

follows assumptions (A1)-(A3) (compare it to the function $\varphi_{1, \gamma}$ defined in (2.3)). Hence the asymptotics of (2.6) follows by Theorem 0.4 , and the proof of the corollary is completed. 
Corollary 2.4. Let $g:[0,1] \rightarrow \mathbb{C}$ be a measurable bounded function admitting the following limit $g(1):=\lim _{r \rightarrow 1-} g(r) \neq 0$. For a $\gamma>0$, we have

$$
\begin{aligned}
& \int_{0}^{1} r^{n} \frac{g(r)}{(1+\log (1 /(1-r)))^{\gamma}} d r=\frac{g(1)}{n(\log n)^{\gamma}}(1+o(1)), \\
& \int_{0}^{1} r^{n} \frac{g(r)}{\left(1+\log _{* k}(1 /(1-r))\right)^{\gamma}} d r=\frac{g(1)}{n\left(\log _{* k} n\right)^{\gamma}}(1+o(1)) .
\end{aligned}
$$

As a concluding remark, we mention that Corollaries 2.3, 2.4 are used in KoitaKupin-Naboko-Touré [5] to compute the spectral asymptotics for special compact Toeplitz operators with non-radial symbols on Bergman space. These results on Toeplitz operators are then applied to the study of banded (Jacobi) matrices, see [5, Lemmae 2.1, 5.1].

Acknowledgments. We would like to thank Leonid Golinskii for helpful discussions. The work is partially supported by the project ANR-18-CE40-0035.

S. Naboko kindly acknowledges the support by RScF-20-11-20032 grant and Knut and Alice Wallenberg Foundation grant. This work was done while S. Naboko's visit to University of Bordeaux in October-November, 2019. He is grateful to the University for the hospitality.

\section{REFERENCES}

[1] Bleistein, N.; Handelsman, R. A. Asymptotic expansions of integrals. Second edition. Dover Publications, Inc., New York, 1986.

[2] Copson, E. T. Asymptotic expansions. Reprint of the 1965 original. Cambridge Tracts in Mathematics, Vol. 55. Cambridge University Press, Cambridge, 2004.

[3] de Bruijn, N. G. Asymptotic methods in analysis. Corrected reprint of the third edition. Dover Publications, Inc., New York, 1981.

[4] Erdélyi, A. Asymptotic expansions. Dover Publications, Inc., New York, 1956.

[5] Koita, M.; Kupin, S.; Naboko, S.; Touré, B. On spectral properties of compact Toeplitz operators on Bergman space with logarithmically decaying symbol and applications to banded matrices, submitted, arxiv: 2006.02586.

[6] Simon, Barry Advanced complex analysis. A Comprehensive Course in Analysis, Part 2B. American Mathematical Society, Providence, RI, 2015.

[7] Watson, G. N. The Harmonic Functions Associated with the Parabolic Cylinder. Proc. London Math. Soc. (2) 17 (1918), 116-148.

[8] Wong, R. Asymptotic approximations of integrals. Corrected reprint of the 1989 original. Classics in Applied Mathematics, Vol. 34. Society for Industrial and Applied Mathematics (SIAM), Philadelphia, PA, 2001.

Institut de Mathématiques De Bordeaux UMR5251, CNRS, Université de Bordeaux, 351 aVe. De la Libération, 33405 Talence Cedex, France

Email address: skupin@math.u-bordeaux.fr

Physics Institute, St. Petersburg State University, Ulyanovskaya str. 1, St. Peterhof, St. Petersburg, 198-904 Russia

Email address: sergey.naboko@gmail.com 